\title{
A NEW HARDENING LAW FOR STRAIN GRADIENT PLASTICITY
}

\author{
S. H. CHEN* and T. C. WANG \\ LNM, Institute of Mechanics, Chinese Academy of Sciences, Beijing 100080, China
}

(Received 5 November 1999; received in revised form 20 June 2000; accepted 10 July 2000 )

\begin{abstract}
A new hardening law of the strain gradient theory is proposed in this paper, which retains the essential structure of the incremental version of conventional $J_{2}$ deformation theory and obeys thermodynamic restrictions. The key feature of the new proposal is that the term of strain gradient plasticity is represented as an internal variable to increase the tangent modulus. This feature which is in contrast to several proposed theories, allows the problem of incremental equilibrium equations to be stated without higher-order stress, higher-order strain rates or extra boundary conditions. The general idea is presented and compared with the theory given by Fleck and Hutchinson (Adv. in Appl. Mech. (1997) 295). The new hardening law is demonstrated by two experimental tests i.e. thin wire torsion and ultra-thin beam bending tests. The present theoretical results agree well with the experiment results. (C) 2000 Acta Metallurgica Inc. Published by Elsevier Science Ltd. All rights reserved.
\end{abstract}

Keywords: Strain gradient plasticity; Microstructure

\section{INTRODUCTION}

Many experiments have shown that materials display strong size effects when the characteristic length scale associated with non-uniform plastic deformation is in the order of microns. For an aluminum-silicon matrix reinforced by silicon carbide particles, Lloyd [13] observed a substantial strength increase when the particle diameter was reduced from 16 to 7.5 microns with the particle volume fraction fixed at $15 \%$. In experiments of measuring micro-indentation hardness of metallic materials, the square of hardness increases linearly as the depth of indentation decreases $[14,15$, $17,19]$. The overall properties of a polycrystal are functions of $l / a$, which reflects the grain size effect, where $l$ is the intrinsic length scale introduced into crystal's constitutive law by the gradient effects and $a$ is the grain size [21]. The most compelling experimental evidence that strong size effects exist have been provided by Fleck et al. [7] and Stolken and Evans [22]. The former is to measure torsion stress strain performed on copper wires, the scaled shear strength increases by a factor of 3 as the thin copper wires diameter decreases from 170 to 12 microns, while the increase of work-hardening in simple tension is negligible. The latter is to bend ultra thin beams and measure the bend moments, a significant increase in the normalized bending hardening is

\footnotetext{
* To whom all correspondence should be addressed.
}

observed as the beam thickness decreases from 50 to 12.5 microns, while data from simple tension displays no size dependence.

The classical plasticity theories can not predict this size dependence of material behavior at the micron scale because their constitutive models possess no internal length scale.

In order to explain the size effect, developing a continuum theory for micron level is needed. Fleck and Hutchinson [5] and Fleck et al. [7] developed a phenomenological theory and a material length scale was introduced for dimensional grounds. From these theoretical developments and consequent attempts at explaining experimental findings of indentation [4, $14,20]$, and fracture [23], it has been found necessary to introduce two length parameters $[4,6]$. One length, $l_{\mathrm{R}}$, refers to rotational gradients as originally proposed in connection with the torsion measurements. The other, $l_{\mathrm{S}}$, refers to stretch gradients. The latter is needed to rationalize length scale phenomena found in indentation and fracture. In 1998, Nix and Gao [18] started from the Taylor relation and gave one kind of hardening law for gradient plasticity. Using the law, they derived the relationship between the indentation hardness $H$ and the depth of indentation, which could show excellent agreement with the experiment results measured by McElhaney et al. [15]. Motivated by the indentation hardening law, Gao et al. [8] proposed a mechanism-based theory of strain gradient plasticity (MSG) based on a multiscale framework linking the 
microscale notion of statistically stored and geometrically necessary dislocations to the mesoscale notion of plastic strain and strain gradient.

All the above strain gradient plasticity theories introduce the higher order stress which is required for this class of strain gradient theories to satisfy the Clausiius-Duhem thermodynamic restrictions on the constitutive model for second deformation gradients $[2,9,10]$. In comparison, no work conjugate of strain gradient has been defined in the alternative gradient theories [3, 16, 24, 25] which represent the strain gradient effects as terms relative with Laplacian of effective strain. Retaining the essential structure of conventional plasticity and obeying thermodynamic restrictions, Acharya and Bassani [1] conclude that the only possible formulation is a flow theory with strain gradient effects represented as an internal variable, which acts to increase the current tangent-hardening modulus. However, there has not been a systematic way of constructing the tangent modulus so as to validate this framework.

In the present paper, the essential structure of the incremental version of conventional $J_{2}$ deformation theory is retained and no work conjugate of strain gradient is defined, the only new formulation is constructing a kind of new hardening law with strain gradient effects as an internal variable which acts to increase the current tangent modulus. It is tested by the wire torsion and ultra-thin beam bending experiment results respectively and compared with the theoretical results obtained from the strain gradient theory proposed by Fleck and Hutchinson [5, 6]. As far as the case of micro-indentation or nano-indentation goes, a detailed analysis will be presented in another paper.

\section{FLECK AND HUTCHINSON'S STRAIN GRADIENT THEORY}

In the theory proposed by Fleck and Hutchinson in 1997, there are three material length scales $l_{1}, l_{2}, l_{3}$, in which the first one is related to the stretch gradient and the other two are related to the rotation gradient. This strain gradient theory is briefly introduced in this section.

Assuming the displacement is $u_{i}$, the strain tensor is $\varepsilon_{i j}$ and the strain gradient is defined as

$$
\eta_{i j k}=u_{k, i j}
$$

The deviatoric part of the strain gradient tensors could be decomposed into three unique, mutually orthogonal third orders deviatoric tensors [20]

$$
\eta_{i j k}^{\prime}=\eta_{i j k}^{(1)}+\eta_{i j k}^{(2)}+\eta_{i j k}^{(3)}
$$

Fleck and Hutchinson [6] introduced the generalized effective strain

$$
E_{e}^{2}=\frac{2}{3} \varepsilon_{i j}^{\prime} \varepsilon_{i j}^{\prime}+l_{1}^{2} \eta_{i j k}^{(1)} \eta_{i j k}^{(1)}+l_{2}^{2} \eta_{i j k}^{(2)} \eta_{i j k}^{(2)}+l_{3}^{2} \eta_{i j k}^{(3)} \eta_{i j k}^{(3)}
$$

In Begley and Hutchinson [4], Equation (3) becomes

$$
E_{e}^{2}=\varepsilon_{e}^{2}+1_{1}^{2} \eta_{i j k}^{(1)} \eta_{i j k}^{(1)}+l_{\mathrm{CS}}^{2} \chi_{e}^{2}
$$

Where

$l_{\mathrm{CS}}^{2}=2 l_{2}^{2}+12 l_{3}^{2} / 5, \varepsilon_{e}=\sqrt{\frac{2}{3} \varepsilon_{i j}^{\prime} \varepsilon_{i j}^{\prime}}, \chi_{i j}=\theta_{i, j}, \chi_{e}=\sqrt{\frac{2}{3} \chi_{i j} \chi_{i j}}$ and $l_{2}=\sqrt{6 / 5} l_{3}$.

Equation (4) can be represented in the following form

$$
\left\{\begin{array}{c}
E_{e}^{2}=\varepsilon_{e}^{2}+l^{2} \eta^{2} \\
\eta=\sqrt{c_{1} \eta_{i j k}^{(1)} \eta_{i j k}^{(1)}+\chi_{e}^{2}}
\end{array}\right.
$$

where $\eta$ is called the effective strain gradient and $l=l_{\mathrm{CS}}, c_{1}=\left(\frac{l_{1}}{l_{\mathrm{CS}}}\right)^{2}$.

The overall stress measure $\Sigma_{e}$ as the work conjugate of $E_{e}$ is

$$
\Sigma_{e}=\frac{d W\left(E_{e}\right)}{d E_{e}}
$$

For the purpose of the following section we shall adopt a simple functional relationship between $\Sigma_{e}$ and $E_{e}$

$$
\Sigma_{e}=A\left(E_{e}\right)
$$

\section{A NEW HARDENING LAW}

The proposed new hardening law of the strain gradient theory preserves the essential structure of the incremental version of conventional $J_{2}$ deformation theory and no extra boundary value conditions beyond the conventional ones are required to solve problems, no higher-order stress or higher-order strain rates are introduced either. The key feature of the new proposal is that the gradient measures explicitly enter the constitutive relations only through the instantaneous tangent modulus and the boundary value problem of incremental equilibrium is the same as in the conventional theories. The tangent hardening modulus is influenced by not only the effective strain but also the effective strain gradient.

In the conventional plasticity theories, $\sigma_{e}$ is the work conjugate of $\varepsilon_{e}$ and defined by

$$
\sigma_{e}=\frac{d W\left(\varepsilon_{e}\right)}{d \varepsilon_{e}}
$$

where $\sigma_{e}=\sqrt{\frac{3}{2} S_{i j} S_{i j}}$ is the usual Von Mises effective stress. 
The work done on the solid per unit volume equals the increment in strain energy

$$
\delta W=S_{i j} \delta \varepsilon_{i j}^{\prime}+\sigma_{m} \delta \varepsilon_{m}
$$

The components $S_{i j}$ of the deviatoric stress tensor can be obtained as

$$
S_{i j}=\frac{2 \sigma_{e}}{3 \varepsilon_{e}} \varepsilon_{i j}
$$

From equation (10), we know that the normality is assumed to be met and there is a yield surface.

The hardening relationship of equation (9) can be expressed as follows

$$
\sigma_{e}=A\left(\varepsilon_{e}\right)
$$

The incremental form of equation (10) can be expressed as

$$
\dot{S}_{i j}=\frac{2 \varepsilon_{i j}}{3 \varepsilon_{e}} \dot{\sigma}_{e}+\frac{2 \dot{\varepsilon}_{i j}}{3 \varepsilon_{e}} \sigma_{e}-\frac{2 \varepsilon_{i j} \sigma_{e}}{3 \varepsilon_{e}^{2}} \dot{\varepsilon}_{e}
$$

and the incremental form of equation (11) is

$$
\dot{\sigma}_{e}=A^{\prime}\left(\varepsilon_{e}\right) \dot{\varepsilon}_{e}
$$

where $A^{\prime}\left(\varepsilon_{e}\right)$ is the tangent hardening modulus in the incremental version of conventional $J_{2}$-deformation theory.

While the strain gradient is considered, the hardening strength is related not only with the density of statistically stored dislocation but also the density of geometrically necessary dislocation. The former is related to the homogeneous deformation $\varepsilon$, the latter with the non-homogeneous deformation and the strain gradient $l \eta$ in a material. We know that when the characteristic length of the deformation field $L$ usually corresponding to the smallest dimension of geometry is much larger than the material length $l$, the strain gradient terms become negligible in comparison with strains, and strain gradient plasticity theory then degenerates to the conventional plasticity theory. However, when $L$ becomes comparable to $l$, strain gradient effects begin to play a dominating role. Instructed by this idea, we propose a new incremental hardening relationship instead of equation (13),

$$
\dot{\sigma}_{e}=A^{\prime}\left(\varepsilon_{e}\right)\left(1+\frac{l^{2} \eta^{2}}{\varepsilon_{e}^{2}}\right)^{\alpha} \dot{\varepsilon}_{e}=B\left(\varepsilon_{e}, l \eta\right) \dot{\varepsilon}_{e}
$$

where $\eta$ is the effective strain gradient defined in equation $(5), B\left(\varepsilon_{e}, l \eta\right)$ is the hardening function including the effect of strain gradient and $\alpha$ is the exponent, in this paper, $\alpha=1$ is taken.
On each incremental step, both the effective strain $\varepsilon_{e}$ and the effective strain gradient $\eta$ can be obtained from the updated displacement fields. Hence $l \eta$ is only a given parameter in equation (14) and it doesn't invoke higher-order stress or higher-order strain rates. The conventional incremental constitutive relation equation (12) is still adaptable in the present theory.

The difference between the present strain gradient theory and the incremental version of conventional $J_{2}$ deformation theory is shown in Appendix A.

The present strain gradient theory can be easily extended to strain gradient flow theory as shown in Appendix B.

\section{COMPARISONS BETWEEN THE THEORETICAL PREDICTIONS AND EXPERIMENT RESULTS}

\subsection{Torsion of thin wires}

A Cartesian coordinate system $\left(x_{1}, x_{2}, x_{3}\right)$ and a cylindrical polar coordinate system $\left(r, \theta, x_{3}\right)$ are introduced as shown in Fig. 1 and $x_{3}$ axis is parallel to the axis of the wire. The radius of the wire is $a . \kappa$ is the twist per unit length of the wire and taken to be positive without loss of generality. Take the displacement field as in classical torsion

$$
u_{1}=-\kappa x_{2} x_{3}, u_{2}=\kappa x_{1} x_{3}, u_{3}=0
$$

and the velocity field is as follows,

$$
v_{1}=-\dot{\kappa} x_{2} x_{3}, v_{2}=\dot{\kappa} x_{1} x_{3}, v_{3}=0
$$

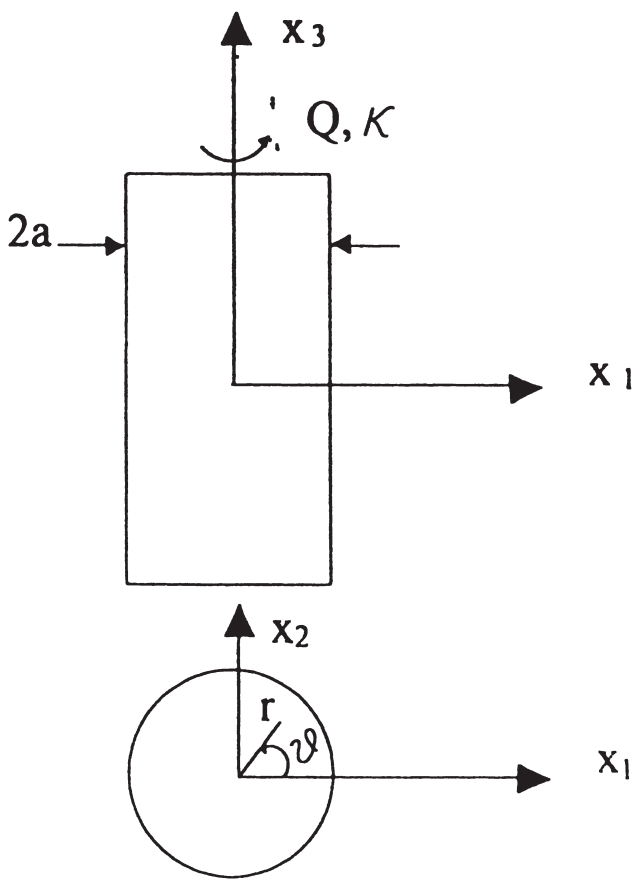

Fig. 1. Plots of two coordinate systems on a thin wire, one is the Cartesian coordinate system $\left(x_{1}, x_{2}, x_{3}\right)$ and the other is the polar coordinate system $\left(r, \theta, x_{3}\right)$. 
The associated non-vanishing components of strain rate and strain are

$$
\left\{\begin{array}{l}
\dot{\varepsilon}_{13}=\dot{\varepsilon}_{31}=-\frac{1}{2} \dot{\kappa} x_{2}, \quad \dot{\varepsilon}_{23}=\dot{\varepsilon}_{32}=\frac{1}{2} \dot{\kappa} x_{1} \\
\varepsilon_{13}=\varepsilon_{31}=-\frac{1}{2} \kappa x_{2}, \quad \varepsilon_{23}=\varepsilon_{32}=\frac{1}{2} \kappa x_{1}
\end{array}\right.
$$

The non-vanishing components of the curvature tensor are

$$
\chi_{11}=\chi_{22}=-\frac{1}{2} \kappa, \chi_{33}=\kappa
$$

then, the effective strain and the effective strain gradient are

$$
\varepsilon_{e}=\frac{1}{\sqrt{3}} \kappa r, \chi_{e}=\kappa
$$

The stretch gradient in equation (3) can be calculated according to Smyshlyaev and Fleck [20]

$$
\eta_{i j k}^{(1)} \eta_{i j k}^{(1)}=0
$$

The generalized effective strain takes the form

$$
E_{e}=\sqrt{\varepsilon_{e}^{2}+l^{2} \chi_{e}^{2}}=\kappa \sqrt{\frac{1}{3} r^{2}+l^{2}}
$$

a) Fleck et al. [7] took a simple power law relationship between the generalized effective stress and effective strain

$$
\Sigma_{e}=\Sigma_{0} E_{e}^{N}=\Sigma_{0}\left(\varepsilon_{e}^{2}+l^{2} \chi_{e}^{2}\right)^{N / 2}
$$

Substituting equations (19) and (22) into the following equation

$$
\int w\left(E_{e}\right) d V=\int_{V}\left(\int_{0}^{E_{e}} \Sigma_{e}\left(E_{e}\right) d E_{e}\right) d V=\int_{0}^{\kappa} Q(\kappa) d \kappa
$$

Finally, the torque given by Fleck et al. [7] is

$$
Q=\frac{6 \pi}{N+3} \sum_{0} \kappa^{N}\left[\left(\frac{1}{3} a^{2}+l^{2}\right)^{(N+3) / 2}-l^{N+3}\right]
$$

From simulating the tensile curves of experiment we take $N=0.22$. If we choose a torsional response curve of $2 a=30 \mu \mathrm{m}$ as a calibration curve, we get $l=3.75 \mu \mathrm{m}$. The comparisons of predicted results with the experiment results are shown in Fig. 2. The theoretical predictions given by the theory of Fleck

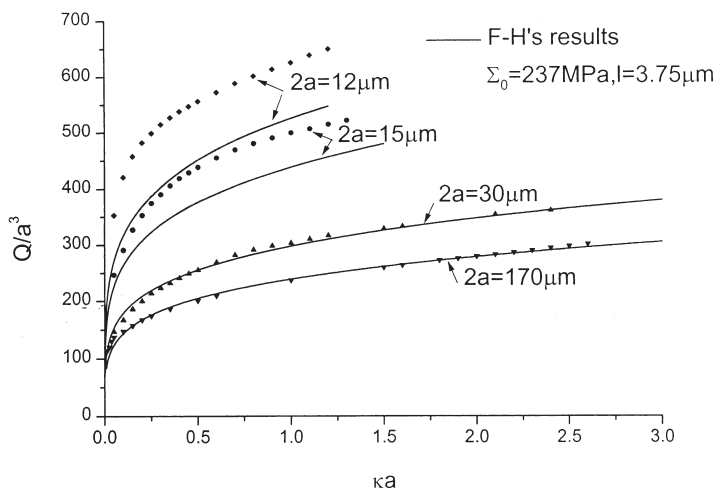

Fig. 2. Plots of torque against the surface strain for copper wires with different diameters. The solid lines denote the theoretical results by Fleck and Hutchinson [6] and the various symbols denote the experiment results [7].

and Hutchinson [6] agree very well with experiment results for the case of $2 a=30 \mu \mathrm{m}$ and $170 \mu \mathrm{m}$. But it seems underestimate the effect of strain gradient on the torsional response for the case of $2 a=15 \mu \mathrm{m}$ and $12 \mu \mathrm{m}$.

b) Now, we use the incremental version of the strain gradient $J_{2}$ deformation theory and the new hardening relationship, equation (14), to investigate the same problem and here take $\alpha=1$. According to equation (22), we have

$$
A\left(\varepsilon_{e}\right)=\sigma_{0} \varepsilon_{e}^{N}, \sigma_{0}=\Sigma_{0}
$$

so equation (14) becomes

$$
\begin{aligned}
& \dot{\sigma}_{e}=N \sigma_{0} \varepsilon_{e}^{N-1}\left(1+\frac{l^{2} \eta^{2}}{\varepsilon_{e}^{2}}\right) \dot{\varepsilon}_{e}= \\
& N \sigma_{0} \varepsilon_{e}^{N-1}\left(1+\frac{3 l^{2}}{r^{2}}\right) \dot{\varepsilon}_{e}
\end{aligned}
$$

From equation (26), we find that the term reflecting the effect of strain gradient has no relation with the deformation history, so for the problem of thin wire torsion, after integrating equation (26) one can obtain following equation,

$$
\sigma_{e}=\sigma_{0} \varepsilon_{e}^{N}\left(1+\frac{3 l^{2}}{r^{2}}\right)
$$

Thus, it is reasonable and convenient to use equation (27) to solve the problem of thin wire torsion.

In this problem, $\eta=\xi_{e}$ and the non-vanishing components of stress can be obtained according to equation (10),

$$
\tau_{13}=\tau_{31}=\frac{2 \varepsilon_{13}}{3 \varepsilon_{e}} \sigma_{e}, \tau_{23}=\tau_{32}=\frac{2 \varepsilon_{23}}{3 \varepsilon_{e}} \sigma_{e}
$$


then the non-vanishing components of stress in the cylindrical coordinate system can be expressed as

$$
\tau_{\theta z}=\frac{1}{\sqrt{3}} \sigma_{e}
$$

Since there is no higher order stresses, the overall torque $Q$ can be obtained from the integration over the cross section of torques produced by the stress components as follows

$$
\begin{gathered}
Q=\int_{0}^{2 \pi} \int_{0}^{a} \tau_{\theta z} r^{2} d r d \theta=\frac{2 \pi a^{3}}{(N+3)(\sqrt{3})^{N+1}} \\
\Sigma_{0}(\kappa a)^{N}\left[1+\frac{3(N+3)}{N+1}\left(\frac{l}{a}\right)^{2}\right]
\end{gathered}
$$

The normalized torque can be written as

$$
\frac{Q}{a^{3}}=\frac{2 \pi \Sigma_{0}}{(N+3)(\sqrt{3})^{N+1}}(\kappa a)^{N}\left[1+\frac{3(N+3)}{N+1}\left(\frac{l}{a}\right)^{2}\right]
$$

The comparisons of equation (31) with test results for copper wires of different diameters are shown in Fig. 3. From Fig. 3 we can find that all curves predicted by equation (31) for different diameters are consistent with the test results and here $l=2.82 \mu \mathrm{m}$.

From equation (29) we know that $\tau_{\theta z}$ is the only non-vanishing component of the stresses and it depends only on $r$. It is easy to verify that all the equilibrium equations are met and the traction free conditions on the lateral boundary of the wire are also satisfied. Hence equation (15) provides a true displacement field for our theory.

\subsection{Bending of thin beams}

In 1998, Stolken and Evans [22] did the bending experiment and observed a strong size effect whereby thin beams display much stronger plastic work hard-

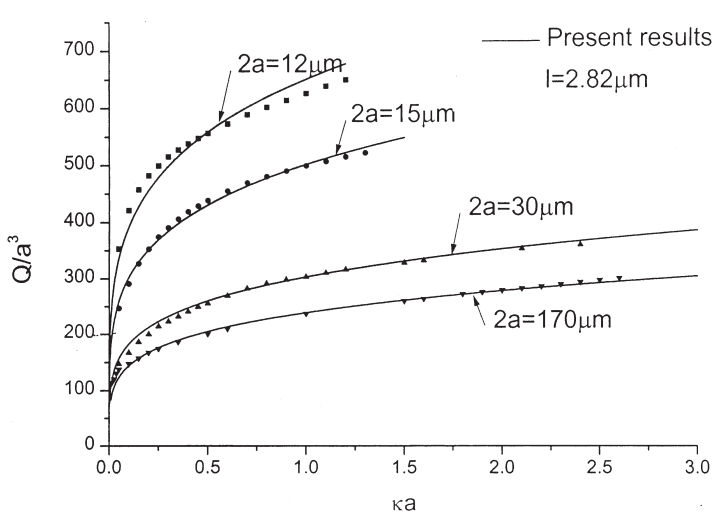

Fig. 3. Plots of torque against the surface strain for copper wires with different diameters. The solid lines denote the theoretical results by the new hardening law proposed in the present paper and the various symbols denote the experiment results [7]. ening than thick ones and no size dependence is observed in the tension test.

Due to the small deformation considered in bending, subject to plane strain deformation, Cartesian $\left(x_{1}\right.$, $\left.x_{2}, x_{3}\right)$ coordinates are adopted as shown in Fig. $4 . \kappa$ is the curvature and $h$ is the beam's thickness. The displacement field is

$$
u_{1}=\kappa x_{1} x_{2}, u_{2}=-\kappa\left(x_{1}^{2}+x_{2}^{2}\right) / 2, u_{3}=0
$$

The velocity field is,

$$
v_{1}=\dot{\kappa} x_{1} x_{2}, \quad v_{2}=-\dot{\kappa}\left(x_{1}^{2}+x_{2}^{2}\right) / 2, v_{3}=0
$$

The non-vanishing strain rates are

$$
\dot{\varepsilon}_{11}=-\dot{\varepsilon}_{22}=\dot{\kappa} x_{2}
$$

The non-vanishing strain components are

$$
\varepsilon_{11}=-\varepsilon_{22}=\kappa x_{2}
$$

The non-vanishing components of curvature tensors are

$$
\chi_{31}=-\kappa
$$

The effective strain and the effective curvature tensor are

$$
\varepsilon_{e}=\frac{2}{\sqrt{3}} \kappa\left|x_{2}\right|, \chi_{e}=\sqrt{\frac{2}{3}} \kappa
$$

The stretch gradient is given by

$$
\eta_{i j k}^{(1)} \eta_{i j k}^{(1)}=\frac{76}{75} \kappa^{2}
$$

We know that the length scale for the stretch gradient is very small and $l_{\mathrm{CR}}$ that corresponds to the rotation gradient is larger [21], then from equation (5), we can find that $c_{1}=\left(\frac{l_{1}}{l_{\mathrm{CR}}}\right)^{2}$ is very small and

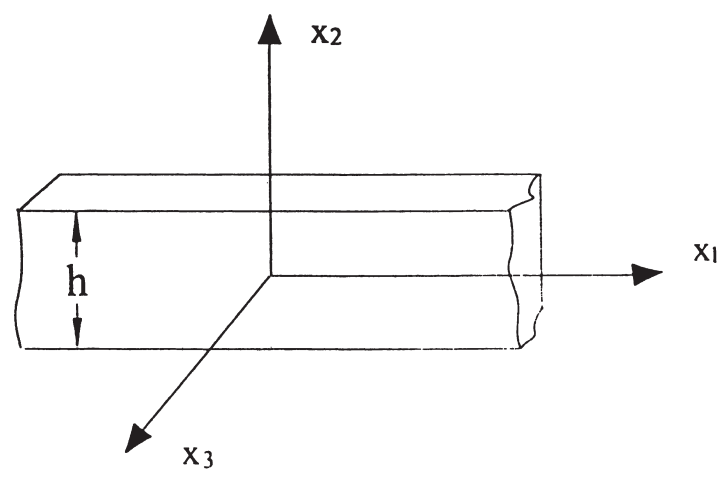

Fig. 4. Plot of coordinate system on the ultra-thin beam used in the present paper. $h$ is the thickness of the beam and the width is taken to be one unit length. 
$\eta_{i j k}^{(1)} \eta_{i j k}^{(1)}$ has the same order as $\chi_{e}^{2}$. In order to be consistent with the theory in analyzing the torsion experiment, we can omit the term of $\eta_{i j k}^{(1)} \eta_{i j k}^{(1)}$ in equation (5) and adopt $\eta=\chi_{e}$, that is only the rotation gradient is considered while investigating the bending experiment.

From simulating the tensile test results of thin beams, the relationship between the stress and plastic strain can be expressed as [22]

$$
\sigma=\Sigma_{0}+\varepsilon_{p l} E_{p}
$$

where $\Sigma_{0}$ is the yield strength, $\varepsilon_{p l}$ is the plastic strain and $E_{p}$ the hardening coefficient. Then the relationship between the effective stress and effective strain can be obtained

$$
\sigma_{e}=\frac{\sqrt{3}}{2} \Sigma_{0}+\frac{3}{4} E_{p} \varepsilon_{e p}
$$

where $\varepsilon_{e p}$ is the effective plastic strain.

a) Stolken and Evans [22] have analyzed this problem based on the strain gradient theory given by Fleck and Hutchinson [6]. The hardening relationship including the effect of strain gradient plasticity is

$$
\begin{aligned}
& \Sigma_{e}=\frac{\sqrt{3}}{2} \Sigma_{0}+\frac{3}{4} E_{p} E_{e}, \\
& E_{e}=\sqrt{\varepsilon_{e}^{2}+l^{2} \eta^{2}}, \\
& \eta=\chi_{e}=\sqrt{\frac{2}{3} \chi_{i j} \chi_{i j}}
\end{aligned}
$$

The strain energy density takes the form

$$
w=\frac{E_{e}}{8}\left(3 E_{p} E_{e}+4 \sqrt{3} \Sigma_{0}\right)
$$

and the total energy per unit length is

$$
W=\int_{-h / 2}^{h / 2} w b d x_{2}
$$

where $b$ is the width of the beam.

Then the bending moment $M$ can be obtained

$$
M=\frac{d W}{d \kappa}
$$

Combining equations (37) and (41)-(44), we obtain

$$
\begin{aligned}
& M=\Sigma_{0} b\left[\frac{h}{4} \sqrt{h^{2}+2 l^{2}}\right. \\
& \left.+\frac{1}{2} l^{2} \ln \left(\frac{\sqrt{h^{2} / 2+l^{2}}+\sqrt{2} h / 2}{l}\right)\right]+
\end{aligned}
$$

$$
\frac{1}{12} E_{p} \kappa b\left(h^{3}+6 l^{2} h\right)
$$

The non-dimensional moment is

$$
\begin{aligned}
& \frac{4 M}{\sum_{0} b h^{2}}=\sqrt{1+2\left(\frac{l}{h}\right)^{2}}+2\left(\frac{l}{h}\right)^{2} \ln \\
& \left(\frac{\sqrt{h^{2} / 2+l^{2}}+\sqrt{2} h / 2}{l}\right) \\
& +\frac{1}{3 \Sigma_{0}} E_{p} \kappa\left(h+6 \frac{l^{2}}{h}\right)
\end{aligned}
$$

The length scale $l$ is determined by fitting equation (46) to all of the bending moment measurements [22]. The comparisons are shown in Fig. 5 with $l=6.12$ $\mu \mathrm{m}$ where $\varepsilon_{b}$ denotes the surface strain.

b) Now, using the new hardening relationship, i.e. equation (14), we investigate the same problem. According to equation (40), the stress-strain curve in uniaxial tensile state can be expressed as

$$
\sigma=\frac{\sqrt{3}}{2} \Sigma_{0}+\frac{3}{4} E_{p} \varepsilon_{p l}
$$

Actually, equation (47) should be represented as follows

$$
\left\{\begin{array}{cc}
\sigma=\hat{\Sigma}_{0}+\hat{E}_{p} \varepsilon & \varepsilon \geq \varepsilon_{0} \\
\sigma=E \varepsilon & \varepsilon \leq \varepsilon_{0}
\end{array}\right.
$$

where $\varepsilon_{0}$ is the yield strain, $\hat{\Sigma}_{0}=\frac{2 \sqrt{3} \Sigma_{0} E}{4 E+3 E_{p}}$ and $\hat{E}_{p}=\frac{3 E_{p} E}{4 E+3 E_{p}}, E=220 \mathrm{GPa}$ is the Young's modulus for $\mathrm{Ni}$.

So the relationship of the effective stress and effective strain is

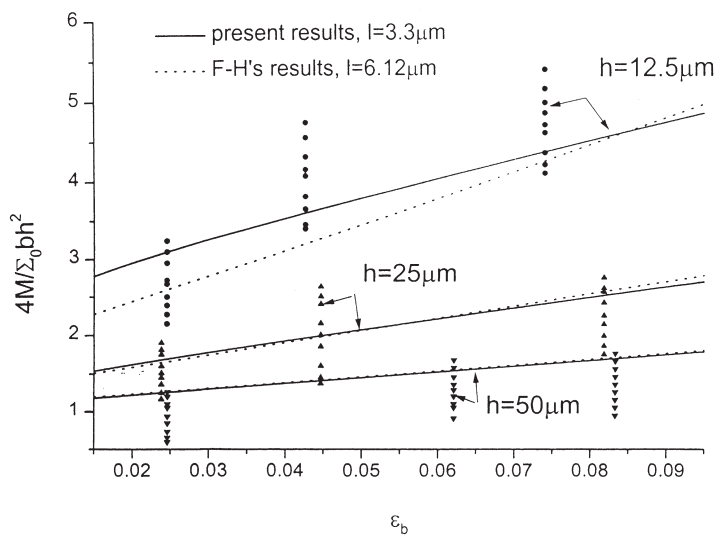

Fig. 5. Plots of bending moment against the surface strain for three beams with different thickness. The dot lines denote the theoretical results by Fleck and Hutchinson [6] and the solid lines denote the present results, the various symbols denote the experiment results [22]. 


$$
\left\{\begin{array}{cc}
\sigma_{e}=\hat{\Sigma}_{0}+\hat{E}_{p} \varepsilon_{e} & \varepsilon_{e} \geq \varepsilon_{0} \\
\sigma_{e}=E \varepsilon_{e} & \varepsilon_{e} \leq \varepsilon_{0}
\end{array}\right.
$$

From equation (47), we can get the yield strength $\sigma_{0}$, which corresponds to the yield strain $\varepsilon_{0}$,

$$
\sigma_{0}=\frac{\sqrt{3}}{2} \Sigma_{0}
$$

then

$$
\varepsilon_{0}=\frac{\sigma_{0}}{E}=\frac{\sqrt{3} \Sigma_{0}}{2 E}
$$

Substituting equations (50) and (51) into equation (49), we find that equation (49) is a continuous function at the point of $\varepsilon_{e}=\varepsilon_{0}$ as shown in Fig. 6.

Considering the strain gradient plasticity, from equations (49) and (14), we can get

$$
\left\{\begin{array}{l}
\dot{\sigma}_{e}=\hat{E}_{p} \dot{\varepsilon}_{e}\left(1+\frac{l^{2} \eta^{2}}{\varepsilon_{e}^{2}}\right) \quad \varepsilon_{e} \geq \varepsilon_{0} \\
\dot{\sigma}_{e}=E \dot{\varepsilon}_{e}\left(1+\frac{l^{2} \eta^{2}}{\varepsilon_{e}^{2}}\right) \quad \varepsilon_{e} \leq \varepsilon_{0}
\end{array}\right.
$$

Substituting equation (37) into equation (52), we obtain

$$
\begin{cases}\dot{\sigma}_{e}=\hat{E}_{p} \dot{\varepsilon}_{e}\left(1+\frac{l^{2}}{2 x_{2}^{2}}\right) & \varepsilon_{e} \geq \varepsilon_{0} \\ \dot{\sigma}_{e}=E \dot{\varepsilon}_{e}\left(1+\frac{l^{2}}{2 x_{2}^{2}}\right) & \varepsilon_{e} \leq \varepsilon_{0}\end{cases}
$$

From equation (53), we find that the term describing the strain gradient effect has no direct relation with the deformation history, so for the problem of ultra-thin beam bending, we can rewrite equation (53) as follows,

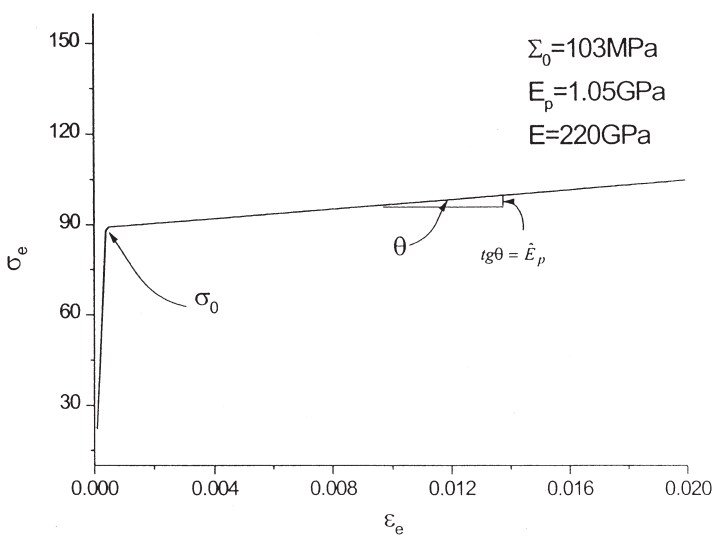

Fig. 6. Plot of relation curve of effective strain and effective stress indicated by Eq. (49), at the point of $\varepsilon=\varepsilon_{0}$, i.e. $\sigma=\sigma_{0}$ the curve is continuous.

$$
\left\{\begin{array}{cc}
\sigma_{e}=\left(\hat{\Sigma}_{0}+\hat{E}_{p} \varepsilon_{e}\right)\left(1+\frac{l^{2}}{2 x_{2}^{2}}\right) & \varepsilon_{e} \geq \varepsilon_{0} \\
\sigma_{e}=E \varepsilon_{e}\left(1+\frac{l^{2}}{2 x_{2}^{2}}\right) & \varepsilon_{e} \leq \varepsilon_{0}
\end{array}\right.
$$

Then it is reasonable and convenient to use equation (54) to solve the problem of ultra-thin beam bending.

Combining equations (10), (35) and (37), we obtain

$$
S_{11}=\frac{\operatorname{sign}\left(x_{2}\right)}{\sqrt{3}} \sigma_{e}
$$

then

$\sigma_{11}=\frac{2 \operatorname{sign}\left(x_{2}\right)}{\sqrt{3}} \sigma_{e}, \sigma_{22}=0, \sigma_{33}=\frac{1}{2} \sigma_{11}, \sigma_{i j}=0(i \neq j)$

The moment $M$ can be obtained from the integration over the cross section by the components of stress as

$$
M=2 \int_{0}^{h / 2} \sigma_{11} b x_{2} d x_{2}
$$

Substituting equations (54) and (56) into equation (57), the moment $M$ can be expressed as

$$
\begin{gathered}
M=\frac{b E \varepsilon_{0}}{\sqrt{3} \kappa^{2}}\left(\varepsilon_{0}^{2}+2 l^{2} \kappa^{2}\right)+\frac{b}{\sqrt{3} \kappa^{2}}\left[\frac { 3 \hat { \Sigma } _ { 0 } } { 2 } \left(\varepsilon_{\max }^{2}\right.\right. \\
\left.-\varepsilon_{0}^{2}\right)+\hat{E}_{p}\left(\varepsilon_{\max }^{3}\right. \\
\left.\left.-\varepsilon_{0}^{3}\right)+2 \hat{\Sigma}_{0} l^{2} \kappa^{2} \ln \frac{\varepsilon_{\max }}{\varepsilon_{0}}+2 \hat{E}_{p} l^{2} \kappa^{2}\left(\varepsilon_{\max }-\varepsilon_{0}\right)\right]
\end{gathered}
$$

where

$$
\varepsilon_{\max }=\frac{\kappa h}{\sqrt{3}}
$$

Comparisons of the theoretical prediction of equation (58) with the bending test results of different thickness are shown in Fig. 5, From Fig. 5 we can find that the calculation results agree well with the test results and the length scale $l=3.3 \mu \mathrm{m}$.

From equation (56), we know that $\sigma_{11}$ and $\sigma_{33}$ are the non-vanishing components of stresses and depend only on $x_{2}$. It is obvious that all the conventional equilibrium equations are met and the traction free conditions on the boundary of the beam are also satisfied. Hence, equation (32) is a true displacement field for our theory. 


\section{CONCLUSION}

The new hardening law of the strain gradient theory outlined in Section 3 retains the algebraic nature of the normality rules of conventional $J_{2}$ deformation theories. There are no higher-order stress, higher-order strain rates or extra boundary conditions introduced for the present strain gradient theory. Only the tangent modulus is changed while considering the material size effects, and the term of strain gradient plasticity is only an internal variable in the hardening law to influence the tangent modulus.

The present paper suggests that the strain gradient strengthening is associated with the ratio of the effective strain gradient to the effective strain. Using the proposed hardening relationship to investigate the problems of thin wire torsion and the micro bending, the theoretical results agree well with the experiment results. The length scale for copper is $l=2.82 \mu \mathrm{m}$ and $l=3.3 \mu \mathrm{m}$ for $\mathrm{Ni}$.

The investigation for micro-indentation or nanoindentation based on the proposed new hardening law is carried on and will be presented in another paper.

Acknowledgements-This work is supported by the National Natural Science Foundation of China (No. 19704100), National Science Foundation of Chinese Academy of Sciences (Project KJ951-1-20), CAS K. C. Wong Post-doctoral Research Award Fund and Post-doctoral Science Funds of China.

\section{APPENDIX A}

a) The conventional $J_{2}$ deformation theory [11, 12] can be expressed as follows. The constitutive relations are

$$
S_{i j}=\Lambda e_{i j}, \sigma_{m}=K \varepsilon_{m}
$$

where

$$
\Lambda=\frac{2 \sigma_{e}}{3 \varepsilon_{e}}
$$

$S_{i j}$ denotes the deviatoric stress, $e_{i j}$ denotes the deviatoric strain, $\sigma_{m}$ is the spherical part of stress and $\varepsilon_{m}$ is the spherical part of strain.

The hardening relationship between the effective stress and effective strain takes the form

$$
\sigma_{e}=A\left(\varepsilon_{e}\right)
$$

Then

$$
\Lambda=\frac{2 \sigma_{e}}{3 \varepsilon_{e}}=\frac{2 A\left(\varepsilon_{e}\right)}{3 \varepsilon_{e}}
$$

From equations (A1) and (A3), the incremental version of conventional $J_{2}$ deformation theory can be obtained

$$
\begin{gathered}
\dot{S}_{i j}=\Lambda \dot{e}_{i j}+\dot{\Lambda} e_{i j}, \dot{\sigma}_{m}=K \dot{\varepsilon}_{m} \\
\dot{\sigma}_{e}=A^{\prime}\left(\varepsilon_{e}\right) \dot{\varepsilon}_{e}
\end{gathered}
$$

where $\Lambda$ can be obtained from the following equation

$$
\dot{\Lambda}=\left[\frac{2}{3} A^{\prime}\left(\varepsilon_{e}\right)-\Lambda\right] \dot{\varepsilon}_{e} / \varepsilon_{e}
$$

b) In the present strain gradient theory, the constitutive relations are the same as that in the conventionally incremental $J_{2}$ deformation theory, i.e. equation (A5). But the increment version of the hardening law between the effective stress and the effective strain is different from the conventional one (i.e. equation (A6)) while considering the strain gradient effect. It means that the $\Lambda$ is different from the conventional one.

From equation (A2), we have

$$
\sigma_{e}=\frac{3}{2} \Lambda \varepsilon_{e}
$$

Combining equations (A8) and (14), we obtain the equation about $\Lambda$ in the present strain gradient theory,

$$
\dot{\sigma}_{e}=\frac{3}{2}\left[\Lambda \dot{\varepsilon}_{e}+\dot{\Lambda} \varepsilon_{e}\right]=B \dot{\varepsilon}_{e}
$$

where $B=A^{\prime}\left(\varepsilon_{e}\right)\left(1+\frac{l^{2} \eta^{2}}{\varepsilon_{e}^{2}}\right)^{\alpha}$.

Then one can easily obtain the following equation

$$
\dot{\Lambda}=\left[\frac{2}{3} B-\Lambda\right] \dot{\varepsilon}_{e} / \varepsilon_{e}
$$

Comparing equations (A7) and (A10), we can find the difference between the incremental version of conventional $J_{2}$ deformation theory and the present strain gradient theory.

\section{APPENDIX B}

As for the flow theory, we can put it conveniently as follows. The relationship of the plastic strain rate and the deviatoric stress for the conventional $J_{2}$ flow theory is

$$
\dot{\varepsilon}_{i j}^{p}=\lambda S_{i j}
$$

where

$$
\lambda=\frac{3 \dot{\varepsilon}_{e}^{p}}{2 \sigma_{e}}
$$


here $\dot{\mathcal{E}}_{e}^{p}$ is the rate of the effective plastic strain and $\dot{\varepsilon}_{e}^{p}=\sqrt{\frac{2}{3} \dot{\varepsilon}_{i j}^{p} \dot{\varepsilon}_{i j}^{p}}$. The relationship of the spherical strain rate and the spherical stress rate is

$$
\dot{\varepsilon}_{m}=\dot{\sigma}_{m} / K
$$

where $K=\frac{E}{1-2 v}, E$ is Young's modulus and $v$ Poisson's ratio.

Considering the elastic strain, the constitutive relationship is

$$
\left\{\begin{array}{c}
\dot{e}_{i j}=\frac{1}{2 \mu} \dot{S}_{i j}+\frac{3 \dot{\varepsilon}_{e}^{p}}{2 \sigma_{e}} S_{i j} \\
\dot{\varepsilon}_{m}=\frac{1-2 v}{E} \dot{\sigma}_{m}
\end{array}\right.
$$

then, we have

$$
\left\{\begin{array}{c}
\dot{S}_{i j}=2 \mu\left(\dot{e}_{i j}-\frac{3 \dot{\varepsilon}_{e}^{p}}{2 \sigma_{e}} S_{i j}\right) \\
\dot{\sigma}_{m}=K \dot{\varepsilon}_{m}
\end{array}\right.
$$

The hardening relation in the conventional $J_{2}$ flow theory is

$$
\dot{\sigma}_{e}=A^{\prime}\left(\varepsilon_{e}^{p}\right) \dot{\varepsilon}_{e}^{p}
$$

The strain gradient flow theory takes the same constitutive equation (B5) as the constitutive equation.

While considering the strain gradient effect, the hardening relation, i.e. equation (14), should be used instead of equation (B6),

$$
\dot{\sigma}_{e}=B\left(\varepsilon_{e}, l \eta\right) \dot{\varepsilon}_{e}
$$

Comparing the conventional flow theory and the strain gradient flow theory, we can find that the difference is only between equations (B6) and (B7).

\section{REFERENCES}

1. Acharya, A. and Bassani, J. L., in Micromechanics of Plasticity and Damage of Multiphase Materials, IUTAM Symposium, Paris, Aug 29-Sept 1, 1995.

2. Acharya, A. and Shawki, T. G. J. Mech. Phys. Solids, 1995, 43, 1751

3. Aifantis, E. C. Trans. ASME J. Eng. Mater. Technol., 1984, 106, 326.

4. Begley, M. R. and Hutchinson, J. W. The mechanics of size-dependent indentation. Harvard University Report Mech 309, Division of Engineering and Applied Sciences, Harvard University, Cambridge, Massachusetts, 1997.

5. Fleck, N. A. and Hutchinson, J. W. J. Mech. Phys. Solids, 1993, 41, 1825.

6. Fleck, N. A. and Hutchinson, J. W., Strain Gradient Plasticity. Advances in Applied Mechanics, ed. J. W. Hutchinson and T. Y. Wu. Academic Press, New York 1997, vol. 33, p. 295.

7. Fleck, N. A., Muller, G. M., Ashby, M. F. and Hutchinson, J. W. Acta Metal. Mater., 1994, 42, 475.

8. Gao, H., Huang, Y., Nix, W. D. and Hutchinson, J. W. J. Mech. Phys. Solids, 1999, 47, 1239.

9. Gurtin, M. E. Arch. Ration. Mech. Anal., 1965, 18, 335.

10. Gurtin, M. E. Arch. Ration. Mech. Anal., 1965, 19, 339.

11. Hill, R. Mathematical Theory of Plasticity, Oxford University Press, Oxford, England 1950.

12. Kachanov, L. M. Foundations of the Theory of Plasticity, North-Holland Publication Company, Amsterdam, London 1971.

13. Lloyd, D. J. Int. Mater. Rev., 1994, 39, 1.

14. Ma, Q. and Clarke, D. R. J. Mat. Res., 1995, 10, 853.

15. McElhaney, K. W., Vlassak, J. J. and Nix, W. D. J. Mat. Res., 1998, 13, 1300.

16. Muhlhaus, H. B. and Aifantis, E. C. Acta Mech., 1991, 89, 217.

17. Nix, W. D. Metall. Trans., 1989, 20A, 2217.

18. Nix, W. D. and Gao, H. J. Mech. Phys. Solids, 1998, 46, 411.

19 Nix, W. D. Mat. Sci. Eng., 1997, A234-236, 37.

20. Poole, W. J., Ashby, M. F. and Fleck, N. A. Scripta Metall. Mat., 1996, 34, 559.

21. Smyshlyaev, V. P. and Fleck, N. A. J. Mech. Phys. Solids, 1996, 44, 465.

22. Stolken, J. S. and Evans, A. G. Acta Mat., 1998, 46, 5109.

23. Wei, Y. and Hutchinson, J. W. J. Mech. Phys. Solids, 1997, 45, 1253.

24. Zbib, H. and Aifantis, E. C., Res. Mech., 1989, 261.

25. Zbib, H. and Aifantis, E. C. Acta Mech., 1992, 92, 209. 